\title{
Cosmological Constraints from the Red-Sequence Cluster Survey
}

\author{
Michael D. Gladders ${ }^{1,2,3}$, H.K.C. Yee ${ }^{1,2,4}$, Subhabrata Majumdar ${ }^{5}$, L. Felipe Barrientos ${ }^{2,6}$, Henk \\ Hoekstra $^{1,7}$, Patrick B. Hall ${ }^{1,2,8}$ and Leopoldo Infante ${ }^{2,6}$
}

\begin{abstract}
We present a first cosmological analysis of a refined cluster catalog from the RedSequence Cluster Survey (RCS). The input cluster sample is derived from 72.07 square degrees of imaging data, and represents a deep uniform subset of the imaging data available within the RCS survey images which probes to the highest redshift and lowest mass limits. The catalog contains 956 clusters over $0.35<z<0.95$, limited by cluster richness and richness error. The calibration of the survey images has been extensively cross-checked against publicly available Sloan Digital Sky Survey imaging, and the cluster redshifts and richnesses that result from this well-calibrated subset of data are robust. We analyze the cluster sample via a general self-calibration technique including scatter in the mass-richness relation, and using reasonable priors on the Hubble constant. We fit simultaneously for the matter density, $\Omega_{m}$, the normalization of the power spectrum, $\sigma_{8}$, and four parameters describing the calibration of cluster richness to mass, its evolution with redshift, and scatter in the richness-mass relation. The principal goal of this general analysis is to establish the consistency (or lack thereof) between the fitted parameters (both cosmological and cluster mass observables) and available results on both from independent measures. From an unconstrained analysis, the derived values of $\Omega_{m}$ and $\sigma_{8}$ are $0.31_{-0.10}^{+0.11}$ and $0.67_{-0.13}^{+0.18}$ respectively. An analysis including Gaussian priors on the slope and zeropoint of the mass-richness relation gives very similar results: $0.30_{-0.11}^{+0.12}$ and $0.70_{-0.15}^{+0.27}$. Both analyses are in acceptable agreement with the current literature. The derived parameters describing the mass-richness relation in the unconstrained fit are also eminently reasonable and in good agreement with existing follow-up data on both the RCS-1 and other cluster samples. Our results directly
\end{abstract}

\footnotetext{
${ }^{1}$ Visiting Astronomer, Canada-France-Hawaii Telescope, which is operated by the National Research Council of Canada, le Centre Nationale de la Recherche Scientifique, and the University of Hawaii.

${ }^{2}$ Visiting Astronomer, Cerro Tololo Inter-American Observatory. CTIO is operated by AURA, Inc. under contract to the National Science Foundation.

${ }^{3}$ The Observatories of the Carnegie Institution of Washington, Pasadena, CA 91101

${ }^{4}$ Department of Astronomy and Astrophysics, University of Toronto, Toronto, ON M5S 3H8, Canada

${ }^{5}$ Canadian Institute for Theoretical Astrophysics, University of Toronto, Toronto, ON M5S 3H8, Canada

${ }^{6}$ Departamento de Astronomía y Astrofísica, Universidad Católica de Chile, Casilla 306, Santiago 22, Chile

${ }^{7}$ Department of Physics and Astronomy, University of Victoria, Victoria, BC V8P 5C2, Canada

${ }^{8}$ Department of Physics and Astronomy, York University, Toronto, ON, M3J 1P3, Canada
} 
demonstrate that future surveys (optical and otherwise), with much larger samples of clusters, can give constraints competitive with other probes of cosmology.

Subject headings: galaxies: clusters: general, cosmological parameters, methods: data analysis

\section{Introduction}

The dependence of the number density of massive clusters with redshift on the cosmological mass density, $\Omega_{m}$, and the normalization of the power spectrum, $\sigma_{8}$, has been noted in the literature for nearly two decades (e.g., Evrard 1989; Oukbir \& Blanchard 1992; Fan, Bahcall \& Cen 1997) and cosmological tests have been attempted using cluster counts over a similar span (e.g., Edge et al. 1990; Oukbir, Bartlett \& Blanchard 1997; Eke et al. 1998; Henry 2000; Bahcall \& Bode 2003; Pierpaoli et al. 2003; Henry 2004). Convergence on the values of $\Omega_{m}$ and particularly on $\sigma_{8}$ has been slow using this general method, though some recent results tend to favor a high normalization, low-density model (e.g., Bahcall \& Bode 2003), irrespective of cosmological constraints from other techniques (e.g., see Spergel et al. 2003, and references therein).

Recently, various extensions of these techniques have been suggested (Haiman, Mohr \& Holder 2001; Levine, Schulz \& White 2002; Hu \& Kravstov 2003; Hu 2003; Majumdar \& Mohr 2003) as a method for measuring the equation of state parameter of dark energy, $w$, and a number of authors have presented parameter accuracy estimates based on future surveys (e.g., Kneissl et al. 2001; Weller et al. 2002; Newman et al. 2002; Weller \& Battye 2003; Majumdar \& Mohr 2004; Wang et al. 2004). Despite this flurry of recent work, little progress has been made in actually confronting the suggested techniques with real cluster survey data, and it remains to be seen whether systematic issues or unconsidered physical effects will limit the utility of cluster mass function cosmological measurements.

In this paper we present a first analysis of a completed cluster survey - the Red-Sequence Cluster Survey (RCS-1; Gladders \& Yee 2005, hereafter GY05) - using the self-calibration technique suggested by Majumdar \& Mohr (2003). Unlike previous analyses of X-ray data (e.g., Bahcall \& Bode 2003; Pierpaoli et al. 2003), the cluster sample used here is drawn from a single homogeneous survey which was executed precisely to enable this type of cosmological analysis, and contains a much larger number of clusters spanning a broad range of mass and redshift.

This paper is organized as follows. In $\S 2$ we describe the input cluster catalog, and extensive tests designed to demonstrate that it is robust and well calibrated. Section 3 describes the methodology of our self-calibration analysis of this catalog, which simultaneously constrains both the cosmology and the mass-observable relation used. In $\S 4$ we describe and discuss the results in the context of current cosmological parameter constraints and our understanding of the mass richness relation in clusters. Our conclusions are summarized in $§ 5$. 


\section{Cluster Catalogs}

The entire RCS-1 survey contains a total of $\sim 90$ degrees of imaging data from both the CanadaFrance-Hawaii Telescope (CFHT) and the Cerro Tololo Inter-American Observatory (CTIO) $4 \mathrm{~m}$ telescope with coverage in both the $R_{C^{-}}$and $z^{\prime}$-band. Exhaustive details of the data acquisition, reduction and analysis and the generation of cluster catalogs from these data are presented in GY05. Some of the data were taken under sub-optimal conditions, with significant transparency and seeing variations, compromising both the image depth and the photometric stability. Based on records from the observing runs, and an examination of the reduced images and catalogs, we have eliminated the poorest data, and restrict the analysis which follows to a survey area of 72.07 square degrees.

The primary parameters of each cluster relevant for the cosmological measurement performed here are the estimated photometric redshift and the cluster richness, which both rely on stable, wellcalibrated photometry for accuracy. In order to check the RCS photometry we have exhaustively compared the portion of the RCS photometric database which overlaps with the most recent Sloan Digital Sky Survey (SDSS; Adelman-McCarthy (2005)) public data release. The overlap consists of 26.29 square degrees, of which 1.44 square degrees is in the CTIO portion of the RCS. We have applied extinction corrections derived from the atlas of Schlegel, Finkbeiner \& Davis (1998) to both the RCS-1 and SDSS magnitudes, and then checked the photometry for stability both at the camera level (i.e., inter-chip) and from pointing-to-pointing and run-to-run. Comparisons in the $z^{\prime}$-band have been made directly; in the $R_{C}$-band we adjust the zeropoints to eliminate the scatter in comparison to the $r^{\prime}$-band, while preserving the mean offset. We have checked and corrected the RCS-1 color terms for each mosaic camera, as well as the zero points for each chip. The corrections are typically a few hundredths of a magnitude, the expected value given the nominal photometric calibration of the survey (GY05).

We have used the bright star and bright galaxy color distributions from each pointing in the RCS-1 to check for color offsets between pointings, in a method similar to that of Hsieh et al. (2005). The comparison of our derived color offsets to direct color corrections derived from comparison to SDSS magnitudes shows this provides accurate colors to better than 0.03 magnitudes. Internally, the color corrections for each pointing deduced from the star and galaxies separately have a scatter of less than 0.025 magnitudes.

The color corrections described above are sufficient to ensure accurate redshifts for RCS1 clusters. However, the richness measurements require well measured magnitudes, and so we have developed a relatively simple correction scheme which uses the counts of faint galaxies in combination with the color corrections. To the precision we require, galaxy counts per pointing should be constant, both because of the large area of each pointing, and because the imaging is

sufficiently deep to probe a large redshift column. A comparison of this simple approach in the regions of SDSS overlap shows that it approximately halves the residual magnitude errors, to about 0.027 magnitudes in each filter per pointing; we have applied this method to all of the data discussed 
here. Further details of this method will be provided elsewhere.

After the procedure described above, the final photometry for the best 72.07 square degrees of the RCS-1 is well calibrated to within a few hundredths of a magnitude. This is sufficient to provide accurate redshifts, with typical uncertainties of less than 0.05 at $z<0.6$, increasing to about 0.09 at $z=1$ (e.g., Gladders 2003), and accurate richnesses (Yee \& López-Cruz 1999). The resulting stability of the magnitudes and counts of galaxies across the entire dataset ensure that the richnesses are systematically stable to well below the random uncertainties which arise predominantly from shot noise in the galaxy counts. The final cluster catalogs based on these data thus have both accurate redshifts and richnesses - precisely the data required to make a cosmological measurement.

\section{Self-Calibration Analysis}

The input to the self-calibration analysis is a catalog of RCS clusters down to a 3.3-sigma significance (GY05) limit. As detailed in GY05 and Gladders \& Yee (2000), this significance is determined from a detailed bootstrap analysis of the non-cluster regions of the RCS-1 survey data directly. At the our chosen 3.3-sigma limit, the contamination of the sample is less than $5 \%$ based on our current understanding of the RCS-1 false-positive rate (Gladders 2001). As in GY05 the nominal lower redshift limit of the catalog is $z=0.2$. However, to avoid a possible Malmquist bias from clusters scattering out of the sample to below the redshift limit of the catalog, we limit our analysis here to clusters at $z>0.35$ (i.e., more than 3 -sigma above the lower redshift limit of the catalog). We also explicitly limit the catalog to $z<0.95$; the detection limit changes rapidly at $z>1$ as the $4000 \AA$ break moves into the $z^{\prime}$-band, and clusters at the highest redshifts require more careful calibration of the photo-z and richness measurements before they can be reliably used for cosmological tests.

We include in our analysis our current understanding of the RCS selection functions (Gladders 2001) with respect to both richness and blue fraction. We take the evolution of the RCS-1 cluster blue fraction with redshift from Loh et al. (2006, in preparation). For all redshifts considered here the completeness corrections are less than $25 \%$, and significantly less than that in all but the highest redshift bin. We have not attempted to model the error on the derived selection functions, leaving that effort to a future paper. Given the magnitude of the corrections however, we do not expect uncertainty in the catalog completeness to be a dominant source of error

We use the richness parameter $B_{g c}$ (Longair \& Seldner 1979) as our estimator for cluster richness. $B_{g c}$ is the amplitude of the cluster-center to galaxy correlation in units of $\left(h_{50}^{-1} \mathrm{Mpc}\right)^{1.77}$ (the fundamental measurement is the evolution-corrected, background-corrected and luminosityfunction normalized overdensity of galaxies within a fixed proper radius of $500 \mathrm{kpc}$, with $h=0.5$ ). $B_{g c}$ has been shown previously to correlate with cluster mass (Yee \& Ellingson 2003; Yee \& LópezCruz 1999). We specifically use the red-sequence richness, $B_{g c R}$ (GY05), in all analyses which 
follow; $B_{g c R}$ is expected to more closely trace the evolution of mass since red cluster galaxies are well established even at $z=1$, and the measurement errors on $B_{g c R}$ are much smaller than on the total richness. In addition to the significance threshold, we limit the input catalog to only clusters with $B_{g c R}>300$, and further limit the catalog to clusters where the error on $B_{g c R}$ is less than $50 \%$. The $B_{g c R}>300$ cut ensures a monotonic mass limit with redshift; the significance cut is insufficient to achieve this since the RCS- 1 data are more sensitive to clusters at moderate redshifts. The limit on richness errors serves to eliminate clusters which are poorly measured due to having only small numbers of galaxies (usually because they are near some artificially shallow portion of the survey data - such as near a bright star). In total the resulting catalog, including both the redshift and richness limits described above, contains 956 clusters. The total number of clusters represented by the catalog, once corrected for incompleteness, is 1086.

The expected surface density of clusters in a solid angle $\Delta \Omega$ at redshift $z$ to a limiting mass $M_{\text {lim }}$ from a fiducial mass function $\frac{d n}{d M}$ is

$$
\frac{d N}{d z}(z)=\Delta \Omega \frac{d V}{d z d \Omega}(z) \int_{M_{l i m}(z)}^{\infty} \frac{d n}{d M} d M
$$

Assuming the data are grouped into redshift bins of width $\Delta z$, the directly observable quantity $N(z)$ will then be given by $N(z)=\int_{z-\Delta z / 2}^{z+\Delta z / 2} \frac{d N}{d z}\left(z^{\prime}\right) d z^{\prime}$. The adopted form of the mass observable relation (used to calculate $M_{l i m}$ ), following Yee \& Ellingson (2003), is given by

$$
M_{200}=10^{A_{B g c}} B_{g c R}^{\alpha}(1+z)^{\gamma},
$$

where $\gamma$ allows for any possible unknown evolution of the mass-richness relation. We use the Jenkins et al. (2001) mass function in our theoretical calculations and a simple NFW profile (Navarro, Frenk \& White 1997) for the dark matter halos to convert from $M_{200}$ to $M_{\text {Jenkins. }}$ Any theoretical uncertainties in the conversion between masses are subsumed in the cluster mass-richness parameter constraints in our self-calibration analysis, described below.

To compare theoretical predictions of the cluster redshift distribution to the RCS-1 input catalog and subsequently estimate the cosmological parameters, we use a Markov-Chain MonteCarlo (MCMC) analysis (S. Majumdar \& G. Cox 2006, in preparation). It has already been demonstrated (Levine, Schulz \& White 2002; Majumdar \& Mohr 2003, 2004; Lima \& Hu 2005) that cluster surveys with thousands of clusters would have enough information (in theory) to simultaneously determine both cosmology and cluster physics, providing a direct measure of the cluster mass-observable relation in addition to cosmological constraints.

Our basic set of parameters consists of 3 cosmological parameters $\left(\Omega_{M}, \sigma_{8}\right.$, and $\left.h\right)$ and 4 cluster parameters. These are the amplitude $A_{B g c}$ and slope $\alpha$ of the mass- $B_{g c R}$ relation, its redshift evolution parameter $\gamma$, and the fractional scatter $f_{s c}$ in the mass- $B_{g c R}$ relation. Due to the exponential sensitivity of the cluster redshift distribution to the underlying cosmology, the 
cluster counts are not only sensitive to the mean richness-mass relation (given by Equation 2) but also to the actual distribution including scatter. We have modeled this by a gaussian scatter parametrized by the mass independent fixed scatter fraction $f_{s c}$. We do not have any implicit redshift dependence on the scatter. The scatter is incorporated by multiplying the mass function with a "selection function" $F(M, z)$ such that

$$
F(M, z)=0.5\left(\operatorname{erf}\left(\frac{M-M_{l i m}(z)}{f_{s c} M_{l i m}(z)}\right)+1\right) .
$$

With the inclusion of the selection function, the lower limit of the integral over mass is changed from $M_{\text {lim }}(z)$ to some $M_{\text {low }}(z)$. For our calculations, we have fixed $M_{l o w}=8 \times 10^{12} M \odot$. For our best fit $M_{\text {lim }}$, the final constraints are not too sensitive to the value of $M_{\text {low }}$ as long as it is $\leq 10^{13} M_{\odot}$. However, we have seen that with the inclusion of scatter one needs to increase the accuracy of numerical integral routines to get convergent $\frac{d N}{d z}$.

Note that from the consideration of cluster counts, the amplitude $A_{B g c}$ and slope $\alpha$ are degenerate (by construction) and the cluster redshift distribution only really constrains the limiting mass $M_{\text {limo }_{0}}$ and its redshift evolution. Hence, we present the basic analysis which follows in terms of $M_{l i m_{0}}$. Results involving $A_{B g c}$ and $\alpha$ are given for comparison with direct cluster observations. This degeneracy between $A_{B g c}-\alpha$ is broken when one takes priors (or a joint analysis) of targeted observations which provide calibrating constraints on cluster mass. A more complex analysis than that presented here which fits the mass function with redshift (rather than its integral) would also in principle break this particular degeneracy. However, such an analysis is not possible with the limited size of the RCS-1 survey.

In our analysis we assume a flat $\Lambda \mathrm{CDM}$ Universe (i.e., $w=-1$ ). Cluster surveys alone cannot constrain the Hubble constant and so we put a Gaussian prior on the Hubble constant (i.e., $h=0.72 \pm 0.08)$. We also fix the spectral index $n_{s}$ and the baryon density $\Omega_{B}$ to first year WMAP values (Spergel et al. 2003). Other parameters are constrained by weak uniform priors, in order to constrain the parameter spaced searched in the MCMC analysis. These various priors are listed in Table 1.

Our MCMC analysis uses the algorithm proposed by Metropolis et al. (1953) to randomly sample the parameter space with a Markov chain whose distribution asymptotically approaches the distribution from which it is being sampled (e.g., Lewis \& Bridle 2002). To construct the chain, we calculate the likelihood at each point in the parameter space under the assumption that the distribution of the clusters in a redshift bin is essentially Poissonian in nature. Our choice of redshiftbin thickness is optimal since the covariance along the $z$-direction is negligible for $\Delta z \sim 0.1$ (Hu \& Cohn 2006). Moreover, the thickness is greater than the redshift uncertainties. Smaller redshift binning would require use of more generalized likelihood functions (Holder 2006; Hu \& Cohn 2006).

Typically we run $\sim 4-6$ chains and need more than half a million points to reach convergence. We have checked that the chains span a large parameter space and sufficiently overlap with each other. We have also seen that inclusion of cluster parameters requires more time for the chains to 
converge than those having no cluster parameters, since cluster parameters significantly widen the parameter space.

\section{Results and Discussion}

\subsection{Analyses Without Mass-Richness Priors}

The principal goal of this paper is to provide a first observational test of whether large cluster surveys - in particular large optical cluster surveys - in combination with a self-calibration analysis can yield useful cosmological constraints. As such we are interested in analyses with only weak priors on the fitted parameters (i.e., the utilitarian uniform priors listed in Table 1), as the consistency of the results to the literature (both cosmologically, and in the cluster-mass observables) yields important insight into the validity of this approach. However, we are ultimately interested in the best possible cosmological constraints from the existing RCS-1 survey data; and so we also include

in the next subsection a preliminary analysis of the cluster sample using Gaussian priors on $A_{B g c}$ and $\alpha$, derived from extant analysis of follow-up studies of the RCS-1 (Blindert et al. 2006, in preparation) and the CNOC-1 survey (Yee \& Ellingson 2003).

The results of our unconstrained analysis are shown in Figures 1-4. Figure 1 shows the input redshift distribution, and the best fit cosmological model. The MCMC analysis has been done in this case with $M_{l i m_{0}}$ as one of the cluster parameters. Figure 2 shows the fully marginalized likelihood distributions for the six fitted parameters in this case, as well as the central values. These values, with $68 \%$ confidence limits, are summarized in Table 2. We show in Figures 3 and 4 the joint likelihood distributions for most parameter pairs. Figure 4 specifically focuses on the relationship between the fractional scatter, $f_{s c}$, and other parameters. The most significant degeneracy, in terms of its impact on the cosmological results, is the relationship between the scatter in the mass-richness relation, and $\sigma_{8}$.

The final values of the cosmological parameters summarized in Table 2 agree well with recent results from the literature. In particular, our result on $\sigma_{8}$ is in good agreement with the recent year-three WMAP constraints $\left(\sigma_{8}\right.$ values range from 0.722 to 0.772 depending on which datasets are analyzed in combination with WMAP; Spergel et al. 2006). Our value of $\Omega_{m}$ similarly agrees with year-three WMAP (Spergel et al. 2006, $\Omega_{m}$ ranges from 0.238-0.266), and spans results from combined analyses of WMAP and the SDSS $(0.30 \pm 0.04$; Tegmark 2004) and WMAP and the $2 \mathrm{dF}$ (0.231 \pm 0.021 ; Cole et al. 2005).

Note that some recent cluster-based results tend to favor higher values of $\sigma_{8}$, which our analysis does not support. These results include studies of aggregate cluster samples selected by other means (e.g., Bahcall \& Bode 2003) as well as the cluster-centric interpretation of the excess small scale power in the CMB in various experiments (Komatsu \& Seljak 2002; Goldstein et al. 2003; Bond et al. 2005). It has been already pointed out by a number of authors (Pierpaoli et al. 2003; Rasia et 
al. 2005) that to get a better handle on $\sigma_{8}$ it is necessary to take into account the uncertainty in the scaling relations, and scatter. As pointed out by Seljak (2002), choice of the normalization of the scaling relation (either observational or from simulations) can give vastly different $\sigma_{8}$; an a priori choice of fixed scaling relation can give tighter yet biased constraints on cosmological parameters. However, a full marginalization over cluster variables is often not done. A comparison of our results to analyses using full marginalization of the cluster scaling relation shows excellent agreement. For example, Pierpaoli et al. (2003) $\sigma_{8}=0.77_{-0.04}^{+0.05}$ and Henry (2004) find $\sigma_{8}=0.66 \pm 0.16$ after marginalizing over the amplitude of mass-observable relation. In comparison to previous works, we not only marginalize over the normalization of the cluster scaling relation but also take into account any uncertainties arising from our incomplete knowledge of the slope, redshift dependence and scatter in the mass-observable relation by marginalizing over these parameters as well.

It is also worth noting two additional points. First, the $\Omega_{m}-\sigma_{8}$ degeneracies are weaker here by comparison to some methods and complementary to the stronger degeneracies seen in current X-ray cosmological constraints using clusters over a smaller redshift baseline (e.g., Pierpaoli et al. 2003), the CMB, or weak lensing (e.g., compare the $\sigma_{8}-\Omega_{m}$ panel of Figure 3 here to Figure 5 in Van Waerbeke, Mellier \& Hoekstra 2005). This is due to the long redshift baseline of the RCS-1 cluster sample. Second, unlike a common tendency in the literature, we report full error bars on parameters (rather than, say, $\sigma_{8}$ at a fixed value of $\Omega_{m}=0.3$ ). Encouragingly, the uncertainty in our constraints on cosmological parameters from the RCS-1 clusters are in excellent agreement with simple 'Fisher-Matrix' forecasts for upcoming large cluster surveys surveys. For, example typical surveys having $10000-20000$ clusters are predicted to constrain $\sigma_{8}$ and $\Omega_{M}$ to 0.07 and 0.03 from $d N / d z$ alone (Majumdar \& Mohr 2004, Wang et al. 2004). RCS-1 with roughly 10 times less clusters gives errors on cosmology which are a factor of 3 larger. This consistency both in central values and uncertainties in $\sigma_{8}$ and $\Omega_{m}$ implies that cluster surveys are on the right track and capable of giving us interesting constraints on cosmological parameters once more clusters are added to the analysis.

The self-calibration approach also provides measures of the mass-observable relation and the evolution of its zero-point with redshift (see Equation 2), as well as the fractional scatter. To make the comparison to current data on the cluster mass-richness relation, we have repeated the analysis of the RCS-1 cluster catalog by replacing $M_{l i m_{0}}$ with the degenerate parameters $A_{B g c}$ and $\alpha$. We find values of $A_{B g c}=10.55_{-1.71}^{+2.27}$ and $\alpha=1.64_{-0.90}^{+0.91}$. These should be compared to the observed value for the CNOC-1 analysis X-ray selected clusters (Yee \& Ellingson 2003), which derived best fits values of $A_{B g c}=9.89 \pm 0.89$ and $\alpha=1.64 \pm 0.28$ (corrected to $h=0.72$, but not corrected for possible evolution as indicated by $\gamma$ ), as well as a recent analysis (Blindert et al. 2006, in preparation) of similar spectroscopy of 33 RCS-1 clusters at median redshift of $z=0.33$, which yields similar values. Furthermore, the fractional scatter in mass observed in the Blindert et al. work is about $70 \%$, in good agreement with our value of $0.73 \pm 0.22$.

That a generalized self-calibration analysis of the RCS-1 optical cluster catalog yields a massrichness relation which is in agreement (in amplitude and slope) with a dynamical study of a 
completely separate X-ray selected cluster sample (Yee \& Ellingson 2003), and (in amplitude, slope, and scatter) with a dynamical study of an intermediate redshift subset of the RCS-1 clusters (Blindert et al. 2006, in preparation), is a significant endorsement of the robustness and reliability of the RCS-1 optical cluster catalog, the self-calibration methodology, and the values of $\Omega_{m}$ and $\sigma_{8}$ derived from these cluster data. Moreover, we note that the mass-richness relation is in excellent agreement with a preliminary analysis of the weak lensing shear from RCS-1 clusters measured with the same survey data (Hoekstra et al., in preparation).

Interpretation of the evolution term in the mass-richness relation is less obvious. Both data (e.g., see Lin \& Mohr 2004, and the discussion therein) and simulations (Zentner et al. 2005) suggest that as much as $50 \%$ of the light in cluster galaxies may be incorporated into the intracluster medium by $z=0$ (and hence would not counted in a richness measure if this intra-cluster light results from the destruction of significant numbers of bright galaxies). Additionally, an analysis of the X-ray temperatures of a small subset of high redshift RCS-1 clusters directly shows that $B_{g c R}$ over-predicts mass by $\sim 1.4$ by $\mathrm{z}=0.8$ compared to $\mathrm{z}=0.2$ (Hicks 2005), though with significant error bars.

The evolution derived in the self-calibration analysis, $0.40_{-3.80}^{+2.11}$, encompasses these results, due to the large uncertainty. In principle we could place priors on the value of $\gamma$ with are significantly smaller than the formal uncertainty. In practice however, we have left $\gamma$ unconstrained; this is done because unresolved evolution in the scatter can show up as a large value of $\gamma$ (the presence of scatter moves the apparent mass limit, and hence evolution in scatter appears as evolution in $\left.M_{\text {Lim }}\right)$. We currently have no direct information on the scatter in the mass-richness relation at $z=1$, and leave $\gamma$ unconstrained to account for this. However, note that the fact that our derived $\gamma$ is small implies that the scatter does not evolve strongly. In situ measurements of mass-observables in a significant number of clusters at $z=1$ are needed to progress further; such work is ongoing now. Also, in larger surveys, the degeneracy with scatter can also be broken by studying the mass function at different redshifts (Lima \& Hu 2005). Even modest scatter in the presence of a steep mass function results in more up-scatter than down-scatter in mass and so the shape of the mass function can be used to calibrate the scatter.

\subsection{Analyses With Mass-Richness Priors}

In order to provide the best possible cosmological constraints from the current cluster sample we have repeated the MCMC analysis using priors on the mass richness relation from both the CNOC-1 dynamical results (Yee \& Ellingson 2003) and those of Blindert et al. on an RCS-1 subsample. The resulting parameter values are summarized in Table 3. The use of external priors significantly reduces errors on the cluster parameters, especially those affecting the limiting mass. Moreover, external priors make the probability distribution of all the cluster parameters more close

to Gaussian, but less so for the cosmological parameters. The priors also have the effect of lowering $M_{\lim 0}$. 
The effect on the derived mean value of the cosmological parameters is remarkably small however, as might be expected given the degeneracies apparent in Figures 3 and 4. Essentially, the degeneracies dominate in the current dataset, and our current observational constraints are insufficiently precise to break those degeneracies. We are unwilling currently to constrain the scatter and redshift evolution using priors based on available data (though such priors would limit the degeneracies currently seen); leaving these parameters free as a check on the consistency of the results is a more robust approach.

\section{Conclusions}

The promise of cluster surveys for cosmology has been long held, but in comparison to other methods (such as weak lensing, the $\mathrm{CMB}$, or $\mathrm{SNe}$ ), little realized. The initial general analysis of the RCS-1 survey presented here is the first ever attempt to use a large homogeneous sample of clusters over a broad mass and redshift range to constrain both $\Omega_{m}$ and $\sigma_{8}$ simultaneously. More importantly, this cluster sample is both optically selected and characterized; the cluster redshifts and mass estimates are derived directly from only two-band imaging to modest depth. It is thus, by comparison to many methods, very modest in terms of the required observational resources.

Using the best available priors on the mass-richness relation, we find $\Omega_{m}=0.30_{-0.11}^{+0.12}$ and $\sigma_{8}=0.70_{-0.15}^{+0.27}$, in excellent agreement with the bulk of the literature (and particularly with the recent year-three WMAP results). Additionally, in an analysis with no priors on the mass-richness relation we find a very similar cosmological result, as well as compelling agreement between our constraints on the amplitude, slope, and scatter in the mass-richness relation and equivalent parameters derived directly from detailed dynamical studies of a sub-sample of the RCS. Our results also compare well with the cluster mass-richness relation from the CNOC-1 cluster sample. The success of the analysis presented here, and the consistency of the results both cosmologically and with our current understanding of cluster observables, demonstrates that both the RCS-1 cluster survey strategy, and the self-calibration methodology are tractable in practice. Perhaps more importantly it also suggests that clusters, at least in aggregate, are well behaved and amenable to being used as cosmological probes to at least $z=1$.

Our self-calibration analysis includes a first effort in treating the scatter in the mass-richness relation, using a fixed, mass-independent fractional scatter about the mean mass-richness relation. We find the scatter to be significantly degenerate with $\sigma_{8}$. These results demonstrate that scatter in the mass-observable relation is clearly an important parameter to consider, and dictate that future efforts to constrain mass-observable relations (regardless of the observable) probe large enough samples with sufficient precision to set significant limits on the scatter, over the entire redshift baseline used in such surveys. While this effect may be mitigated using mass observables with intrinsically smaller scatter, direct in situ determination of the scatter will remain an important component of upcoming large surveys. Detailed follow-up study of a subsample of the RCS-1 cluster catalogs with this in mind, as well as the imaging for the more than ten times larger RCS-2 survey, 
is ongoing; further cosmological results from these data will be presented in future papers.

Overall, the results shown here hold great promise for much larger optical surveys now underway, ongoing and proposed X-ray surveys, as well as large Sunyaev-Z'eldovich effect-based cluster surveys which will begin soon. The analysis presented here is relatively straightforward; additional leverage on cosmological constraints in larger surveys can be derived from inclusion of, for example, the cluster power-spectrum. Whether or not these additional gains will need to be surrendered to a more complex treatment of the mass-observable scatter is not yet clear, but the good agreement between our results and current predictions of uncertainties in these large surveys is a positive first step towards realizing the promise of galaxy clusters as a precision cosmological probe.

M.D.G. acknowledges previous support from the Natural Sciences and Engineering Research Council of Canada (NSERC) via a post-doctoral fellowship. M.D.G. also acknowledges useful discussions with Dick Bond, Wayne Hu, David Spergel, Martin White, Joe Mohr, and John Carlstrom. S.M. would like to acknowledge Joe Mohr, Martin White and Dick Bond for discussions on cluster surveys and Graham Cox for summer work on MCMC code. The MCMC runs were carried out on the CITA Beowulf cluster and used the publicly available getdist package for analysis work. The RCS-1 project is supported by grants from the Canada Research Chair Program, NSERC, and the University of Toronto to H.K.C.Y. This research as been partially supported by FONDECYT under proyecto 1040423 and Centro de Astrofisica FONDAP. We thank Kris Blindert for sharing the results of her analysis of the mass-richness relation in intermediate redshift RCS-1 clusters in advance of publication, and similarly thank Yeong Loh for the results on the RCS-1 cluster blue fractions.

Facilities: CFHT(CFH12K), CTIO(MOSAIC-II).

\section{REFERENCES}

Adelman-McCarthy, J.K. et al. 2005, ApJS, submitted, astro-ph/0507711

Bahcall, N.A., \& Bode, P. 2003, ApJ, 588, 1

Bond, J.R. et al. 2005, ApJ, 262, 12

Cole, S., et al. 2005, MNRAS, 362, 505

Edge, A.C., Stewart, G.C., Fabian, A.C., \& Arnaud, K.A. 1990, MNRAS, 245, 559

Eke, V.R., Cole, S., Frenk, C.S., \& Henry, P.J. 1998, MNRAS, 298, 1145

Evrard, A.E. 1989, ApJ, 341, 71

Fan, X., Bahcall, N.A., \& Cen, R. 1997, ApJ, 490, 123

Gladders, M. D., \& Yee, H. K. C. 2000, AJ, 120, 2148 
Gladders, M.D. 2001, PhD. Thesis, U Toronto

Gladders, M.D. 2003, in Carnegie Observatories Astrophysics Series Vol. 3, Clusters of Galaxies: Probes of Cosmological Structure and Galaxy Evolution, eds. J. S. Mulchaey, A. Dressler, \& A. Oemler (Cambridge: Cambridge Univ. Press), 89

Gladders, M.D., \& Yee, H.K.C. 2005, ApJ, 157, 1

Goldstein, J.H. et al. 2003, ApJ, 599, 773

Haiman, Z., Mohr, J.J., \& Holder, G.P. 2001, ApJ, 553, 545

Henry, P.J. 2000, ApJ, 534, 565

Henry, P.J. 2004, ApJ, 609, 603

Hicks, A.K. 2005, Ph.D. Thesis, U. of Colorado

Holder, G.P. 2006, astro-ph/0602251

Hsieh, B.C., Yee, H.K.C., Lin, H., \& Gladders, M.D. 2005, ApJS, 158, 161

Hu, W. 2003, Phys. Rev. D, 67, 081304

Hu, W. \& Cohn, J.D. 2006, astro-ph/0602147

Hu, W., \& Kravtsov, A.V. 2003, ApJ, 584, 702

Jenkins, A., et al. 2001, MNRAS, 321, 372

Kneissl, R. et al. 2001, MNRAS, 328, 783

Komatsu, E., \& Seljak, U. 2002, MNRAS, 336, 1256

Levine, E.S., Schulz, A.E., \& White, M. 2002 ApJ, 577, 569

Lewis, A., \& Bridle, S. 2002, Phys. Rev. D, 66, 3511

Lima, M., \& Hu, W. 2005, Phys. Rev. D, 72, 3006

Lin, Y.-T., \& Mohr J.J. 2004, ApJ, 617, 879

Longair, M. S., \& Seldner, M. 1979, MNRAS, 189, 433

Majumdar, S., \& Mohr, J.J. 2003, ApJ, 585, 603

Majumdar, S., \& Mohr, J.J. 2004, ApJ, 613, 41

Metropolis, N., Rosenbluth, A.W., Rosenbluth, M.N., Teller, A.H., \& Teller, E. 1953, Journal of Chemical Physics, 21, 1087

Navarro, J.F., Frenk, C.S., \& White, S.D.M. 1997, ApJ, 490, 493

Newman, J.A., Marinoni, C., Coil, A.L., \& Davis, M. 2002, PASP, 114, 29 
Oukbir, J., Bartlett, J.G., \& Blanchard, A. 1997, A\&A, 320, 365

Oukbir, J., \& Blanchard, A. 1992, A\&A, 262, 21

Pierpaoli, E., Borgani, S., Scott, D., \& White, M. 2003, MNRAS, 342, 163

Rasia, E., Mazzotta, P., Borgani, S., Moscardini, L., Dolag, K., Tormen, G., Diaferio, A., \& Murante, G. 2005, ApJ, 618, L1

Schlegel, D.J., Finkbeiner, D.P., \& Davis, M. 1998, ApJ, 500, 525

Seljak, U. 2002, MNRAS, 337, 769

Spergel, D.N. et al. 2003, ApJS, 148, 175

Spergel, D.N. et al. 2006, astro-ph/0603449

Tegmark, M. 2004, Phys. Rev. D, 69, 10351

Wang, S., Khoury, J., Haiman, Z., \& May, M. 2004, PhRvD, 70, 123008

Weller, J., Battye, R.A., \& Kneissl, R. 2002, Phys. Rev. Lett., 88, 231301

Weller, J., \& Battye, R.A. 2003, NewAR, 47, 775

Van Waerbeke, L., Mellier, Y., \& Hoekstra, H. 2005, A\&A, 429, 75

Yee, H.K.C., \& Ellingson, E. 2003, ApJ, 585, 215

Yee, H.K.C., \& López-Cruz, O. 1999, AJ, 117, 1985

Zentner, A.R., Berlind, A.A., Bullock, J.S., Kravtsov, A.V., \& Wechsler, R.H. 2005, ApJ, 624, 505 


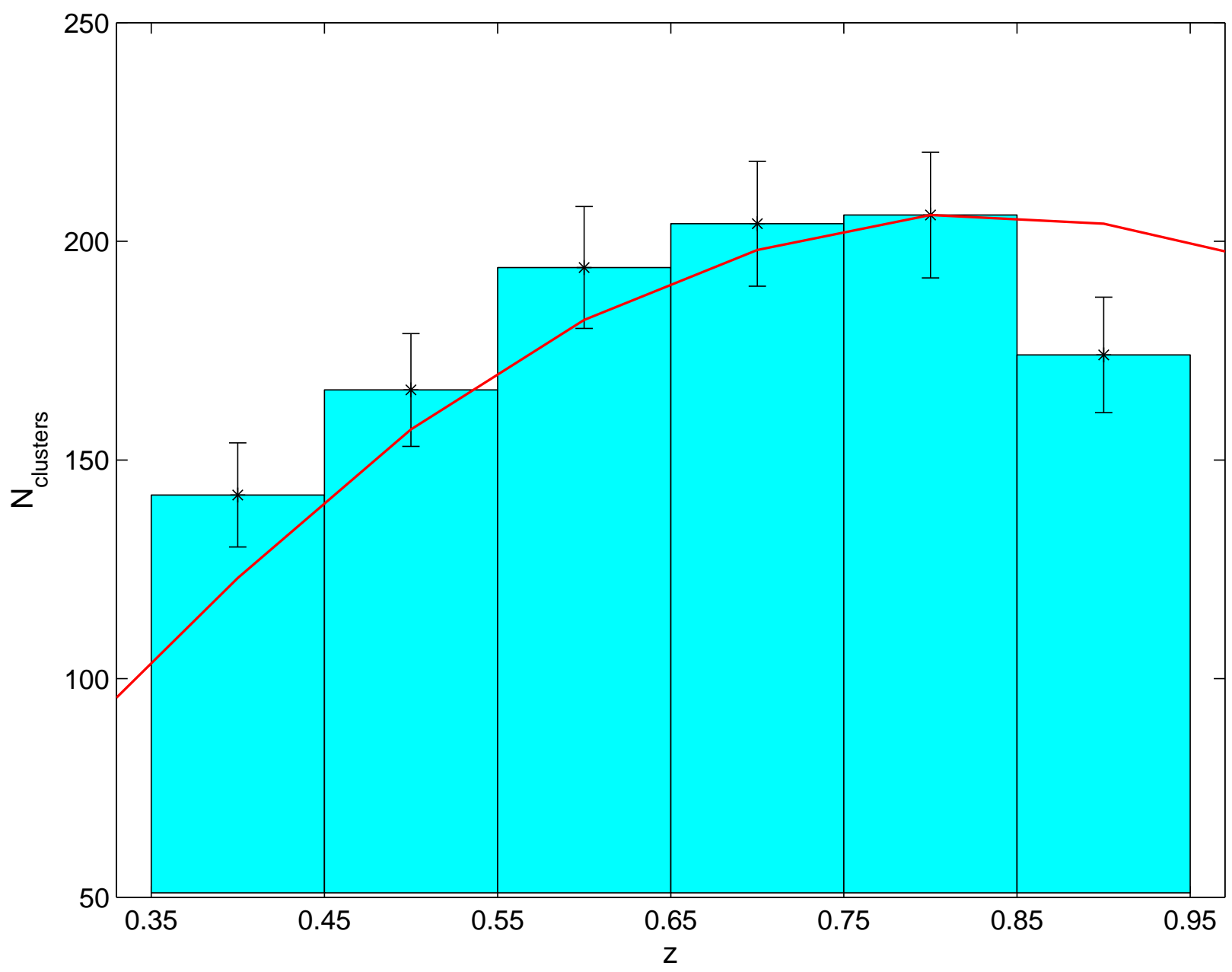

Fig. 1. - The input redshift distribution (histogram), along with the best fit cosmological model (solid line). Error bars are Poisson only. 

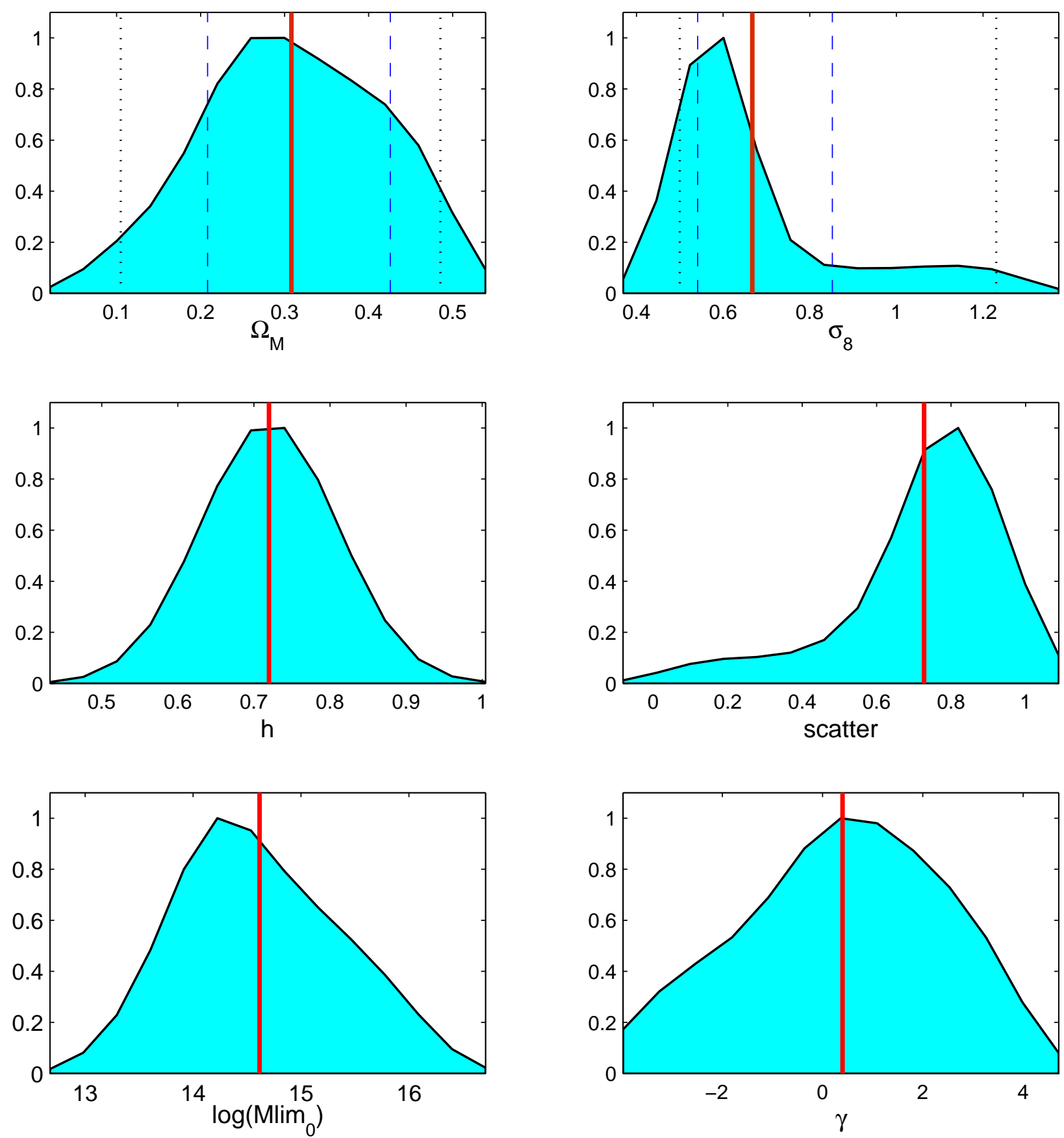

Fig. 2.- Likelihood functions of the RCS-1 cluster $\frac{d N}{d z}$ data as function of 6 basic parameters used in generating the Markov Chains. There are 3 cosmological parameters $\left(\Omega_{m}, \sigma_{8}\right.$, and $\left.h\right)$ and 3 cluster parameters $\left(M_{l i m_{0}}, \gamma\right.$, and $\left.f_{s c}\right)$. The shaded region shows the marginalized likelihood from the Markov chains. The marginalized likelihoods are very non-Gaussian for all cases (as expected for cluster counts) except that of $h$ where a Gaussian prior is used. Notice the long tails in $\sigma_{8}$ and $f_{s c}$. The mean value for each parameter is shown by the solid line. For $\Omega_{M}$ and $\sigma_{8}$ we also show the $1-$ and $2-\sigma$ regions by the dashed and dotted lines respectively. 

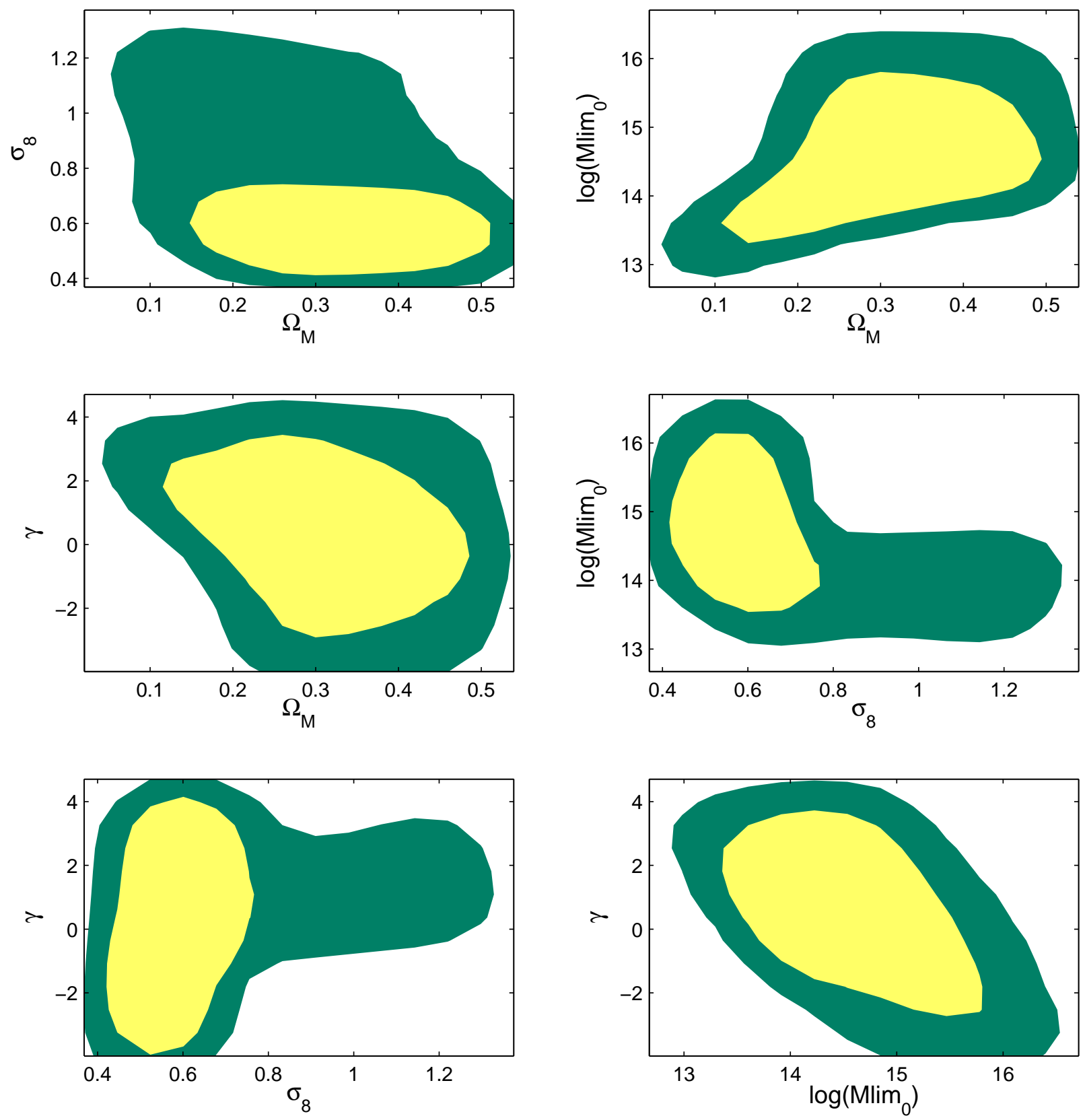

Fig. 3.- The shaded regions show the joint 1 and $2 \sigma$ confidence regions for cosmological parameters $\Omega_{M}$ and $\sigma_{8}$ and cluster parameters $M_{l i m_{0}}$ and $\gamma$ which dictates the mass limit of the survey at any redshift. Additional constraints from targeted observations of clusters can break the current degeneracies. 

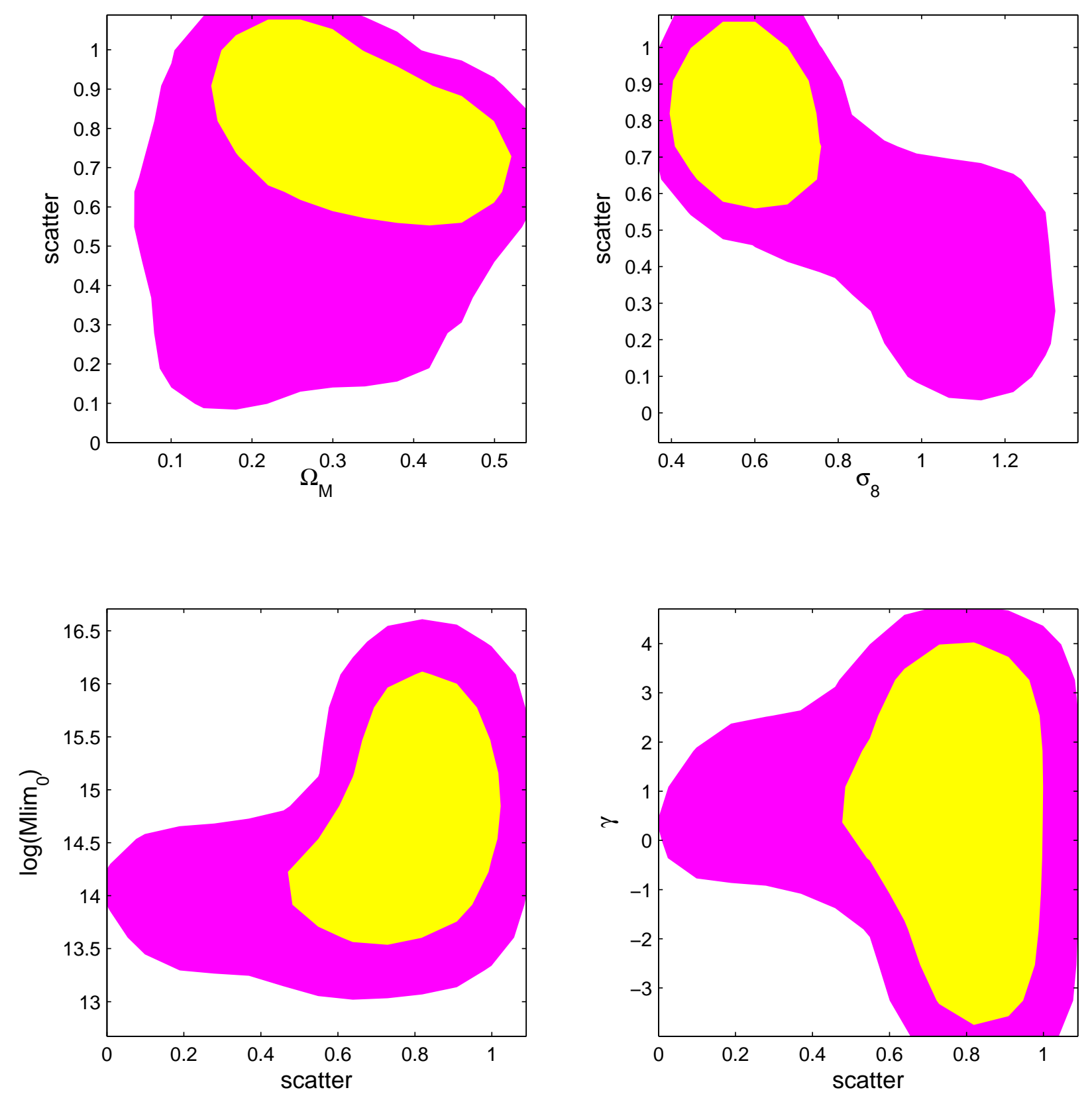

Fig. 4.- The importance of scatter in RCS-1 constraints. The shaded regions show the joint 1 and $2 \sigma$ confidence regions of $\Omega_{M}, \sigma_{8}$, and $M_{l i m_{0}}$ with $f_{s c}$. The scatter and its dispersion comes out of the self-calibration analysis of the RCS-1 clusters and are in good agreement with targeted observations of a subsample of RCS-1 clusters at moderate redshifts. 
Table 1: Priors in MCMC Analysis.

\begin{tabular}{ccl}
\hline Parameter & Prior & Notes \\
\hline$\Omega_{m}$ & $0.05-0.55$ & uniform \\
$\sigma_{8}$ & $0.40-1.30$ & uniform \\
$h$ & $0.72 \pm 0.08$ & Gaussian \\
$\Omega_{b}$ & 0.046 & fixed \\
$n$ & 0.99 & fixed \\
& & \\
\hline$A_{B g c}$ & $6-14$ & uniform \\
$\alpha$ & $0-3$ & uniform \\
$\gamma$ & $-4-4$ & uniform \\
$f_{s c}$ & $0-1$ & uniform \\
\hline
\end{tabular}

Table 2: Derived Parameters from the Self-Calibration Analysis without Mass-Richness Priors.

\begin{tabular}{cc}
\hline Parameter & Mean $(68 \%$ Confidence Range) \\
\hline$\Omega_{m}$ & $0.31_{-0.10}^{+0.11}$ \\
$\sigma_{8}$ & $0.67_{-0.13}^{+0.18}$ \\
\hline $\log \left(M_{\text {lim }}\right)$ & $14.61_{-0.70}^{+0.82}$ \\
$\gamma$ & $0.40_{-3.80}^{+2.11}$ \\
$f_{s c}$ & $0.73_{-0.16}^{+0.18}$ \\
\hline
\end{tabular}

Table 3: Derived Parameters from the Self-Calibration Analysis with Mass-Richness Priors.

\begin{tabular}{ccc}
\hline Parameter $^{\mathrm{a}}$ & $\begin{array}{c}\text { Mean (68\% Confidence Range) } \\
\text { (Blindert } \text { et al. 2006 Priors) }\end{array}$ & $\begin{array}{c}\text { Mean (68\% Confidence Range) } \\
\text { (Yee \& Ellingson 2003 Priors) }\end{array}$ \\
\hline$\Omega_{m}$ & $0.30_{-0.11}^{+0.12}$ & $0.31_{-0.10}^{+0.11}$ \\
$\sigma_{8}$ & $0.70_{-0.15}^{+0.27}$ & $0.68_{-0.14}^{+0.22}$ \\
\hline$A_{B g c}$ & $9.61_{-0.65}^{+0.65}$ & $10.27_{-0.66}^{+0.67}$ \\
$\alpha$ & $1.92_{-0.24}^{+0.24}$ & $1.70_{-0.24}^{+0.24}$ \\
$\gamma$ & $0.81_{-1.61}^{+1.66}$ & $0.64_{-1.90}^{+1.96}$ \\
$f_{s c}$ & $0.69_{-0.20}^{+0.20}$ & $0.71_{-0.17}^{+0.19}$ \\
\hline
\end{tabular}

\footnotetext{
${ }^{a}$ For the case with no priors, parameter values are as reported in Table 2, with values of $A_{B g c}$ and $\alpha$ as reported in
} the main text $\left(A_{B g c}=10.55_{-1.71}^{+2.27}\right.$ and $\left.\alpha=1.64_{-0.90}^{+0.91}\right)$. 